\title{
Determinants of difficulty in activities of daily living in ambulatory patients undergoing hemodialysis
}

Takaaki Watanabe', Toshiki Kutsuna ${ }^{1,2}$, Kei Yoneki ${ }^{1}$, Manae Harada', Takahiro Shimoda', Yusuke Matsunaga ${ }^{3}$, Norio Murayama ${ }^{4}$, Ryota Matsuzawa ${ }^{5}$, Yasuo Takeuchi ${ }^{6}$, Atsushi Yoshida ${ }^{7}$ and Atsuhiko Matsunaga ${ }^{1 *}$

\begin{abstract}
Background: Patients undergoing hemodialysis (HD) have difficulty performing activities of daily living (ADL) compared to healthy people. ADL difficulty is an early predictor of loss of independence and mortality in older community-living people. However, determinants of ADL difficulty in HD patients have not been clarified. This study aimed to identify factors associated with ADL difficulty in ambulatory HD patients.
\end{abstract}

Methods: Subjects were 216 Japanese outpatients (130 men, 86 women; mean age, 67 years) undergoing maintenance HD three times a week. Clinical characteristics, depressive symptoms, motor function (leg strength, balance, and walking speed), and ADL difficulty related to lower-limb function such as mobility issues were compared across three difficulty levels (higher, middle, and lower) as classified according to the percentages of patients with perceived difficulty. Multivariate logistic regression analysis was performed to examine whether clinical characteristics, depressive symptoms, and motor function could discriminate ADL difficulty at each level. Receiver operating characteristic curve analysis was performed to determine cut-off values of motor function for predicting ADL difficulty at each level.

Results: $A D L$ difficulty was independently associated with age (odds ratio $(\mathrm{OR})=1.05,95 \%$ confidence interval $(\mathrm{Cl}) 1$. $00-1.10 ; P=0.039)$, presence of depressive symptoms $(\mathrm{OR}=4.24,95 \% \mathrm{Cl} 1.13-15.95 ; P=0.033)$, and usual walking speed $(\mathrm{OR}=0.94,95 \% \mathrm{Cl} 0.90-0.97 ; P<0.001)$ for higher level difficulty; age $(\mathrm{OR}=1.06,95 \% \mathrm{Cl} 1.02-1.10 ; P=0.006)$, maximum leg strength $(\mathrm{OR}=0.97,95 \% \mathrm{Cl} 0.94-1.00 ; P=0.043)$, and usual walking speed $(\mathrm{OR}=0.96,95 \% \mathrm{Cl} 0.93-0.98 ; P=0.001)$ for middle level difficulty; and age ( $\mathrm{OR}=1.06,95 \% \mathrm{Cl} 1.02-1.10 ; P=0.006)$ and usual walking speed $(\mathrm{OR}=0.93,95 \% \mathrm{Cl} 0.90-0$. 6; $P<0.001)$ for lower level difficulty. Cut-off values of usual walking speed for predicting ADL difficulty for higher, middle, and lower level difficulty were $83.7,75.5$, and $75.1 \mathrm{~m} / \mathrm{min}$, respectively.

Conclusions: A slow walking speed and old age were significantly and independently associated with ADL difficulty in ambulatory HD patients. Presence of depressive symptoms was significantly and independently associated with ADL difficulty at the higher level of difficulty in ambulatory HD patients. These findings provide useful data for planning effective therapeutic regimens to prevent ADL difficulty in ambulatory HD patients.

Keywords: Activities of daily living (ADL) difficulty, Depressive symptom, Walking speed, Hemodialysis patient

\footnotetext{
* Correspondence: atsuhikonet@gmail.com

${ }^{1}$ Department of Rehabilitation Sciences, Kitasato University Graduate School of Medical Sciences, 1-15-1 Kitasato, Sagamihara, Kanagawa 252-0373, Japan Full list of author information is available at the end of the article
} 


\section{Background}

Evaluation of physical functioning in maintenance hemodialysis (HD) patients is recommended by the Kidney Disease Outcomes Quality Initiative clinical practice guidelines [1] and European Renal Best Practice guidelines [2], as decreased physical functioning leads to a reduced quality of daily life and higher risk of mortality in this patient population [1-4]. These guidelines recommend assessment of physical functioning using both self-reported and openfield measures of physical performance $[2,5,6]$. In particular, self-reported measures of physical functioning have the advantage of being simple and easy to use [2].

In Japan, more than half of HD patients reportedly have the ability to perform basic activities of daily living (ADL) and instrumental ADL tasks without assistance [7]. However, even HD patients who can perform ADL tasks independently show high rates of ADL difficulty, i.e., difficulty carrying out routine self-care activities. Kutner et al. [8] reported that $\mathrm{HD}$ patients are more likely to have ADL difficulty compared to age-matched controls. Moreover, HD patients have been reported to show poor physical functioning (e.g., leg strength, standing balance, and walking ability), with high rates of depressive symptoms [9-13]. Recent studies have shown that poor standing balance, slow walking speed, presence of depression, and chronic kidney disease predict ADL difficulty in older community-living people [14-17]. Moreover, ADL difficulty has been shown to be an early predictor of loss of independence and mortality in older community-living people [18]. However, few studies have examined factors associated with ADL difficulty in HD patients. Therefore, this study aimed to identify factors associated with ADL difficulty in clinically stable ambulatory $\mathrm{HD}$ patients.

\section{Methods}

\section{Study population}

The study protocol was approved by the Ethics Committee of Kitasato University. Informed consent was obtained from each patient after providing a detailed explanation of the study protocol. From July 2007 to October 2016, clinically stable outpatients at the Sagami Circulatory Organ Clinic Hemodialysis Center were assessed for eligibility for inclusion in this cross-sectional study. Patients were undergoing maintenance HD therapy three times a week according to the Japanese Society for Dialysis Therapy guidelines. Patients were excluded if they fulfilled the following criteria: hospitalization $\leq 3$ months prior to study enrollment; presence of uncontrolled cardiac arrhythmias, severe disdialysis syndrome (hypotension, nausea, and muscle spasm during hemodialysis), peripheral artery disease with apparent intermittent claudication or critical limb ischemia, chronic heart failure (New York Heart Association classes III to IV), or uncontrolled hypertension (blood pressure at rest > 180/110 $\mathrm{mmHg}$ ); need for walking assistance; and presence of any other conditions that limited walking (e.g., dementia, low vision or blindness, paralysis due to stroke, and leg amputation).

\section{Clinical characteristics}

Information regarding age, sex, HD duration, body mass index (BMI), primary end-stage renal disease, and laboratory values (blood hemoglobin and serum albumin) was obtained from clinical records. BMI was calculated by dividing weight in kilograms by the square of height in meters. Blood hemoglobin and serum albumin concentrations were measured immediately before each hemodialysis session.

Presence of diabetes mellitus, cerebrovascular disease (stroke), and cardiac disease (angina pectoris, myocardial infarction, percutaneous coronary intervention, or coronary artery bypass grafting) was assessed based on clinical records. Moreover, to quantify comorbid illnesses, we used a comorbidity index developed for dialysis patients, which comprised the following primary causes of kidney disease: atherosclerotic heart disease, congestive heart failure, cerebrovascular accident/transient ischemic attack, peripheral vascular disease, dysrhythmia, and other cardiac diseases; chronic obstructive pulmonary disease; gastrointestinal bleeding; liver disease; cancer; and diabetes [19].

\section{Depressive symptoms}

A short, 10-item version of the Center for Epidemiological Studies Depression Screening Index (CES-D) was used to assess depressive symptoms in the last 7 days $[13,20,21]$. Each response item is rated on a scale ranging from 0 to 3 . Total CES-D score was calculated as the sum of all item scores and ranged from 0 to 30 . Higher scores indicated greater depressive symptoms, and the presence of depressive symptoms was defined as a score of $\geq 10$ [21].

\section{Motor function}

Assessment of motor function included measurements of leg strength, standing balance, and walking ability. Leg strength was evaluated using a handheld dynamometer ( $\mu$ tas F-1; Anima, Tokyo, Japan). Patients were asked to sit on a bench with their hip and knee flexed at an angle of $90^{\circ}$, and then, the maximum voluntary isometric knee extensor strength was measured three times. Maximum leg strength was expressed as a percentage of body weight, i.e., the average of right and left maximum isometric leg strength divided by weight $[22,23]$.

Standing balance was evaluated by measuring one-leg standing time. The duration that patients could stand on one leg with their eyes open, while holding their hands at their waist without any aid or falling, was measured using a stopwatch [24]. The measurement was performed for up 
to $60 \mathrm{~s}$ and stopped if patients hopped, stepped, put the raised foot down on the other foot or on the floor, or released their hands from the waist. Patients underwent a second trial if they were unable to stand on one leg for $60 \mathrm{~s}$ in the first trial [25]. The maximum time of the two measurements was used for analysis.

Walking ability was evaluated by measuring usual walking speed along a $10-\mathrm{m}$ walkway. Usual walking speed was defined as distance $(10-\mathrm{m})$ divided by time (in minutes) from self-selected walking speed [22]. Usual walking speed was measured once and was expressed in meter per minutes.

\section{ADL dependency}

ADL dependency was assessed by the Functional Independence Measure (FIM). The FIM is widely used to measure patient functional status for performing ADL. In the USA, the FIM has been used as a primary outcome measure and, approximately, $60 \%$ of US hospitals used the FIM in 2002 [26]. The FIM comprises 13 motor items and five cognitive items, which are rated on a 7-point scale $(1$, complete assistance, to 7 , complete independence) $[27,28]$. In this study, only five motor items (transfer to bed, chair, or wheelchair; transfer to toilet; transfer to tub or shower; walking or wheelchair propulsion; and stair climbing) related to lower-limb function were used, with a total score of 7 to 35 points. Lower scores indicated greater ADL dependency.

\section{ADL difficulty}

ADL difficulty was assessed with a questionnaire developed for patients undergoing HD therapy to assess their perceived difficulty in performing ADL related to lower-limb function such as mobility. This questionnaire on perceived mobility difficulty for HD patients comprises 12 items divided into the following three categories, which were obtained by a factor analysis: "basic ADL," "ambulation," and "walking up or down stairs" [29, 30]. The reliability and validity of the questionnaire have been assessed in Japanese HD patients [29, 30]. The English version of this questionnaire was created through a process of repeated translation from Japanese to English by a bilingual physiotherapist (A.M.) and then back-translated by a bilingual translator.

Additional file 1: Table S1 shows the 12 items of the questionnaire. Patients were asked to rate their perceived difficulty in performing these items on a scale of 1 to 5 ( 1 , not possible; 2 , severe difficulty; 3 , moderate difficulty; 4, mild difficulty; and 5, ease). ADL difficulty score was defined as the sum of all points and ranged from 12 to 60 points. Lower scores indicated greater ADL difficulty.

\section{Statistical analysis}

To compare score distribution, histograms of ADL dependency and difficulty scores from all patients were created at 5-point intervals. According to a method described previously [29], the 12 items of the ADL difficulty questionnaire were classified into three difficulty levels (higher, middle, and lower). Then, patients with a perceived difficulty score $\leq 3$ for at least one item in each level were allocated into the difficulty group, and other patients (perceived difficulty score $\geq 4$ for all items) were allocated into the non-difficulty group. Differences in clinical characteristics (age, sex, BMI, HD duration, primary cause of end-stage renal disease, hemoglobin, serum albumin, and comorbid conditions), depressive symptoms, motor function (maximum leg strength, one-leg standing time, and usual walking speed), and ADL dependency and difficulty scores between the two groups were assessed for significance using the non-paired $t$ test and $\chi^{2}$ test. Univariate and multivariate logistic regression analyses were performed to assess whether clinical characteristics and motor function could discriminate the presence of ADL difficulty for each level. Independent variables used for multivariate logistic regression analysis were age, sex, blood hemoglobin concentration, serum albumin concentration, comorbidity index, depression symptoms, maximum leg strength, one-leg standing time, and usual walking speed for each level. Receiver operating characteristic (ROC) curve analysis was performed to determine cut-off values of motor function for predicting ADL difficulty at each level. Cut-off values were determined according the maximum Youden index [31].

All analyses were performed using the Statistical Package for Social Sciences (IBM SPSS Statistics 24.0 for Windows; IBM Corp., Armonk, NY, USA). $P<0.05$ was considered statistically significant.

\section{Results \\ Patient characteristics, motor function, ADL dependency, and ADL difficulty}

As shown in Fig. 1, 199 of 564 Japanese HD outpatients who were assessed for eligibility fell under the exclusion criteria, and 149 declined to participate. Consequently, 216 patients ( 130 men, 86 women) were included in this study.

Additional file 2: Table S2 shows the clinical characteristics, motor function, ADL dependency, and ADL difficulty of all 216 patients (mean age, 67.4 years; age range, 36-90 years). The most common underlying kidney disease was glomerulonephritis (35.2\%), followed by diabetic nephropathy (31.9\%). Mean ADL dependency and difficulty scores were $34.3 \pm 0.9$ and $43.6 \pm 10.6$ points, respectively. 


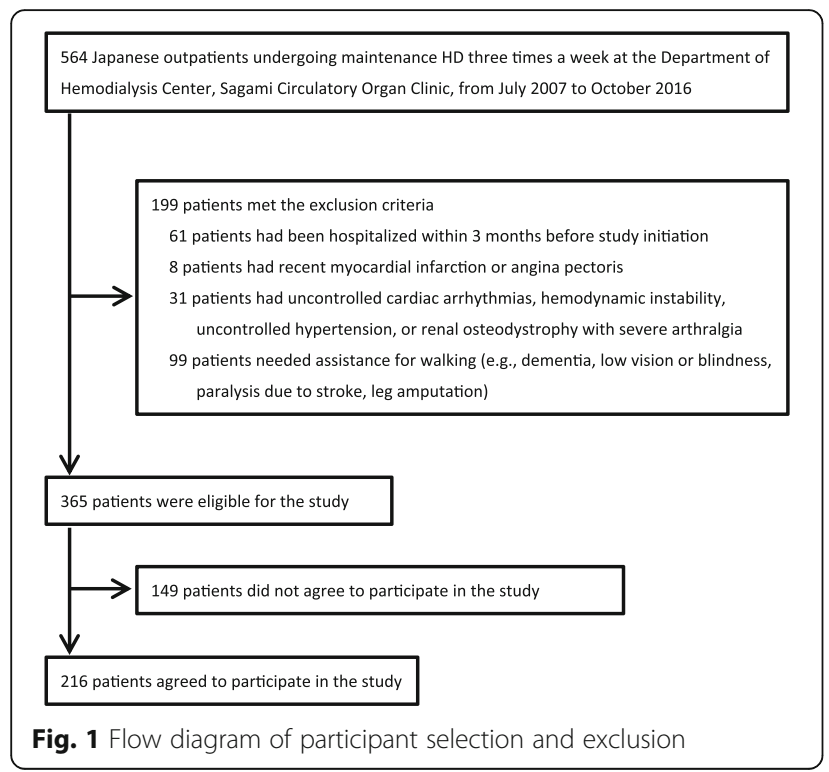

Figure 2a shows the distribution of ADL dependency scores. Fifty-two percent (113 persons) scored full points. Figure $2 \mathrm{~b}$ displays the distribution of ADL difficulty scores, showing wide variation. Four percent (nine persons) scored full points.

As shown in Fig. 3, patients found "walking up two flights of stairs" the most difficult of all 12 items, followed by "walking $1 \mathrm{~km}$," "walking $600 \mathrm{~m}$," "rising from the floor," "walking down two flights of stairs," "walking $300 \mathrm{~m}$," "walking up one flight of stairs," "sitting down on the floor," "walking 20 m quickly," "walking down one flight of stairs," "walking $100 \mathrm{~m}$," and "rising from a chair." Furthermore, based on the percentages of patients with a perceived difficulty score $\leq 3$, "walking up two flights of stairs," "walking $1 \mathrm{~km}$," "walking $600 \mathrm{~m}$," and "rising from the floor" were classified as ADL items with higher level difficulty; "walking down two flights of stairs," "walking 300 m," "walking up one flight of stairs," and "sitting down on the floor" were classified as ADL items with middle level difficulty; and "walking 20 m quickly," "walking down one flight of stairs," "walking $100 \mathrm{~m}$," and "rising from a chair" were classified as ADL items with lower level difficulty.

Differences in clinical characteristics, motor function, depressive symptoms, ADL dependency score, and ADL difficulty score between the difficulty and non-difficulty groups for each difficulty level are shown in Additional file 2: Table $\mathrm{S} 2$. For higher level difficulty, significant differences were observed between the two groups in age $(P<0.001)$, presence of depressive symptoms $(P=0.002)$, maximum leg strength $(P<0.001)$, one-leg standing time $(P<0.001)$, and usual walking speed $(P<0.001)$. For middle level difficulty, significant differences were observed in age $(P<0.001)$, presence of depressive symptoms $(P=0.001)$, maximum leg

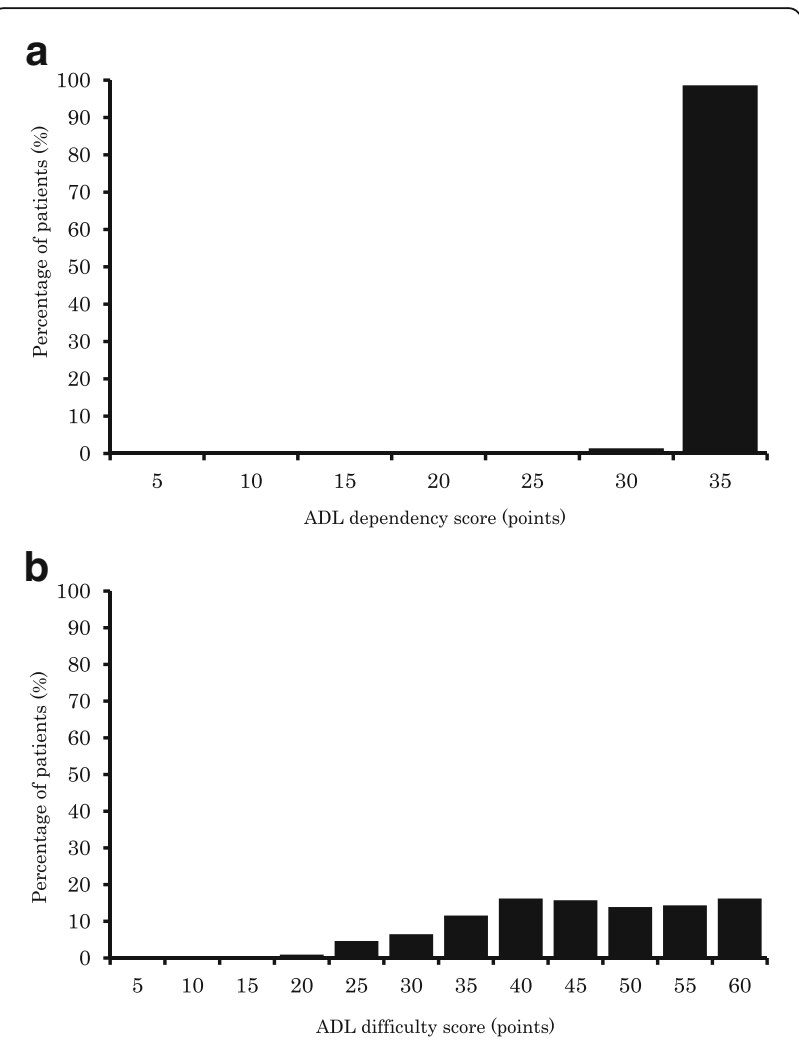

Fig. 2 Histogram of ADL dependency and ADL difficulty scores. a ADL dependency was assessed by the FIM. b ADL difficulty was assessed by the questionnaire on perceived mobility difficulty. $A D L$, activities of daily living; FIM, functional independence measure

strength $(P<0.001)$, one-leg standing time $(P<0.001)$, and usual walking speed $(P<0.001)$. For lower level difficulty, significant differences were observed in age $(P<0.001)$, maximum leg strength $(P<0.001)$, one-leg standing time $(P<0.001)$, and usual walking speed $(P<0.001)$.

\section{Univariate and multivariate logistic regression analyses on ADL difficulty}

The results of univariate and multivariate logistic regression analyses on ADL difficulty are shown in Additional file 3: Table S3. As shown in Additional file 3: Table S3, the results of multivariate logistic regression analysis on ADL difficulty revealed that presence of depressive symptoms (odds ratio $(\mathrm{OR})=4.03,95 \%$ confidence interval $(\mathrm{CI}) 1.12-14.50$; $P=0.033)$ and usual walking speed $(\mathrm{OR}=0.94,95 \% \mathrm{CI}$ 0.90-0.97; $P<0.001$ ), after adjusting for age and sex (model $1)$, and presence of depressive symptoms (OR $=4.24$, 95\%CI 1.13-15.95; $P=0.033$ ) and usual walking speed (OR $=0.94,95 \% \mathrm{CI} 0.90-0.97 ; P<0.001)$, after adjusting for model 1 plus hemoglobin, serum albumin, and comorbidity index, were significantly associated with ADL difficulty at the higher level of difficulty. At the middle level of difficulty, maximum leg strength (OR $=0.97,95 \% \mathrm{CI}$ 0.94-1.00; $P=0.043)$ and usual walking speed $(\mathrm{OR}=0.96,95 \% \mathrm{CI}$ 


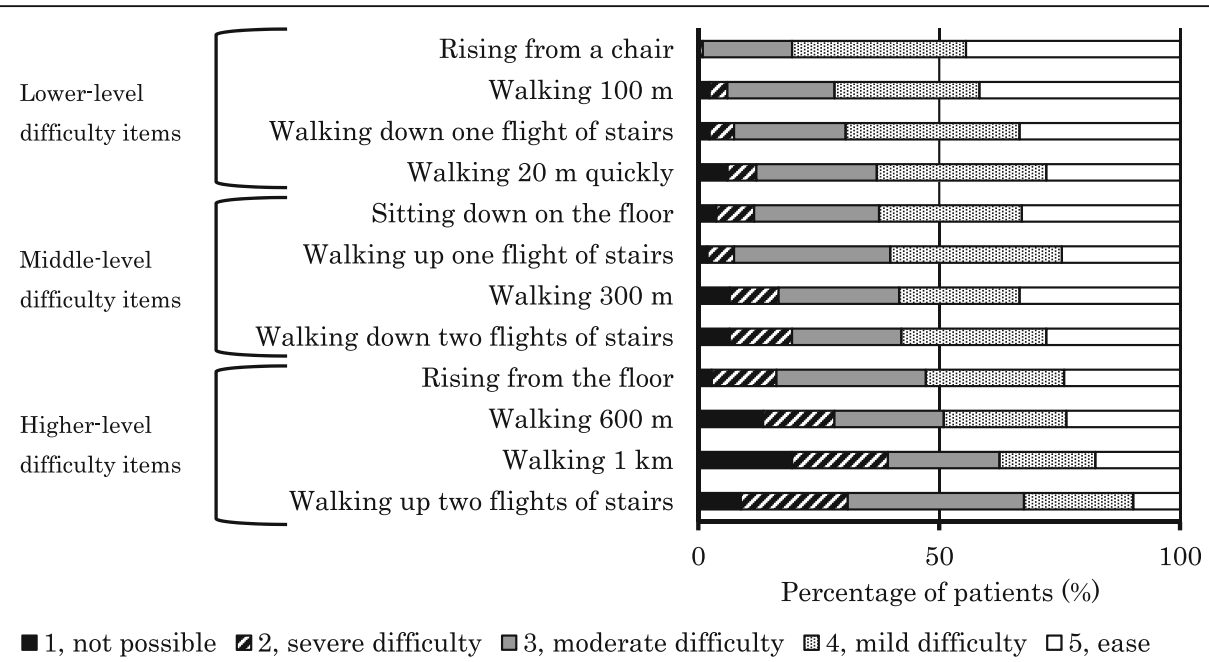

Fig. 3 Difficulty levels and distribution of 12 items of the questionnaire on perceived mobility difficulty

0.93-0.98; $P=0.001)$, in model 1 , and maximum leg strength $(\mathrm{OR}=0.97,95 \% \mathrm{CI} 0.94-1.00 ; P=0.043)$ and usual walking speed $(\mathrm{OR}=0.96,95 \% \mathrm{CI} 0.93-0.98 ; P=0.001)$, in model 2, were significantly associated with ADL difficulty. At the lower level of difficulty, usual walking speed (OR = 0.93, 95\%CI 0.90-0.96; $P<0.001$ ), in model 1 , and usual walking speed $(\mathrm{OR}=0.93$, 95\%CI $0.90-0.96 ; \mathrm{P}<0.001)$, in model 2 , were significantly associated with ADL difficulty.

\section{ROC curves and cut-off values of usual walking speed for predicting ADL difficulty}

Since usual walking speed was significantly associated with ADL difficulty across all three levels, ROC curves were generated for usual walking speed. Figures 4, 5, and 6 show the ROC curves of usual walking speed for predicting ADL difficulty at each level. The areas under the ROC curves of usual walking speed for predicting ADL difficulty at higher, middle, and lower levels were 0.82 $(P<0.001), 0.78(P<0.001)$, and $0.82(P<0.001)$, respectively. Cut-off values of usual walking speed for predicting $\mathrm{ADL}$ difficulty at higher, middle, and lower levels were $83.7,75.5$, and $75.1 \mathrm{~m} / \mathrm{min}$, respectively.

\section{Discussion}

In the present cross-sectional study, ADL difficulty scores varied widely among $\mathrm{HD}$ patients, whereas more than half of $\mathrm{HD}$ patients scored full points for $\mathrm{ADL}$ dependency. We classified the 12 items of the ADL difficulty questionnaire into three difficulty levels (higher, middle, and lower) based on the percentages of patients with a perceived difficulty score $\leq 3$ in order to identify determinants of ADL difficulty. Multivariate logistic regression analysis revealed that common factors independently associated with ADL difficulty were old age and low usual walking speed for all difficulty levels, presence of depressive symptoms for higher level difficulty, and decreased maximum leg strength for middle level difficulty. Cut-off values of usual walking speed for predicting the presence of ADL difficulty for higher, middle, and lower level difficulty were 83.7, 75.5, and $75.1 \mathrm{~m} / \mathrm{min}$, respectively.

HD patients reportedly have a significantly higher ADL difficulty score compared to healthy controls [8]. Patients hospitalized due to heart failure have also been shown to have difficulty in walking (29.0\%) and climbing stairs (45.9\%) [32]. In the present study, 61 (28.2\%) and $86(39.8 \%)$ patients reported difficulty in walking $100 \mathrm{~m}$

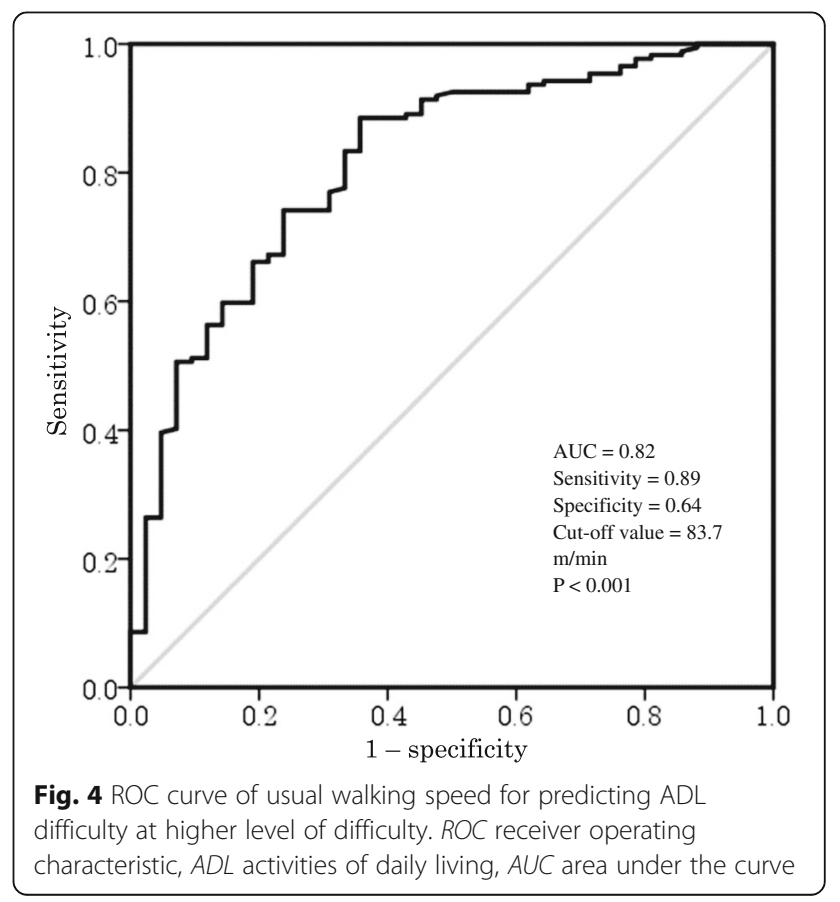




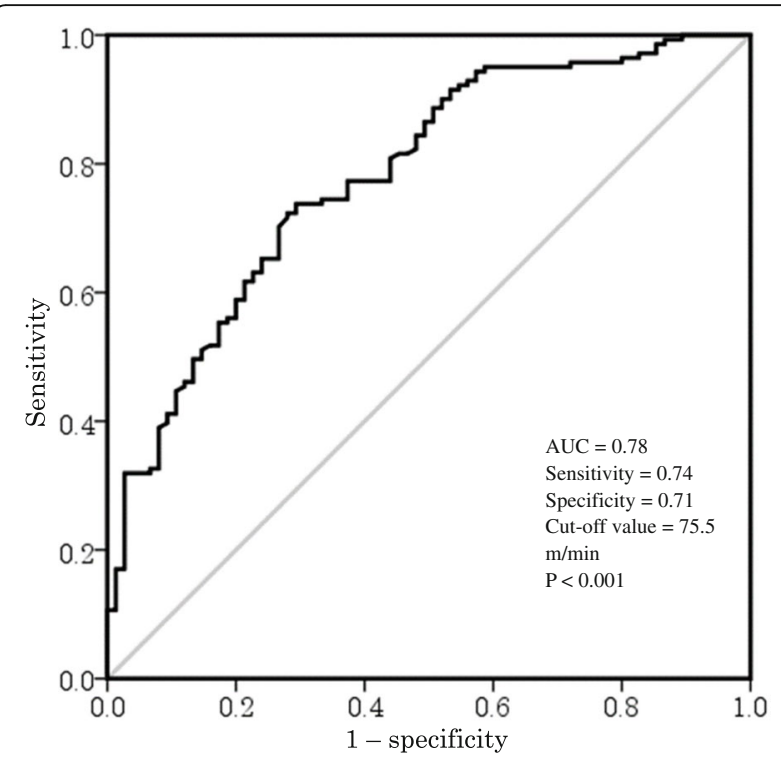

Fig. 5 ROC curve of usual walking speed for predicting ADL difficulty at middle level of difficulty. $R O C$ receiver operating characteristic, $A D L$ activities of daily living, AUC area under the curve

and walking up one flight of stairs, respectively, suggesting that prevalence rates of reported difficulty are similar between HD patients and hospitalized patients with heart failure.

In this study, usual walking speed was significantly associated with ADL difficulty at all three levels after adjustment for confounders. In a previous cross-sectional study, walking speed was associated with ADL difficulty

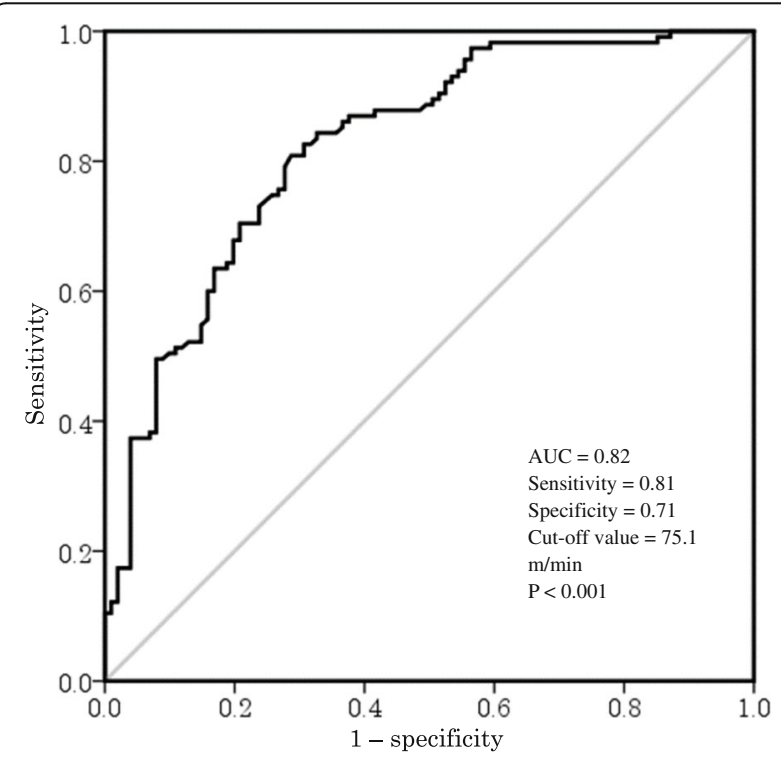

Fig. 6 ROC curve of usual walking speed for predicting $A D L$ difficulty at lower level of difficulty. $R O C$ receiver operating characteristic, ADL activities of daily living, AUC area under the curve in community-dwelling adults aged 65 and older [33]. Moreover, an 18-month prospective study reported that walking speed can predict the onset of ADL difficulty in older women aged 70-80 years [14]. Our findings are in line with these reports.

The presence of depressive symptoms was significantly associated with ADL difficulty at the higher level of difficulty. Depression has been attributed to disabilities in basic ADL (e.g., inability to bathe, eat, dress, transfer from a bed to a chair, use the toilet, or walk across a small room), mobility (e.g., unable to walk a short distance without help, or walking up and down stairs without help), and limited social activity (e.g., social withdrawal or social isolation) in older communitydwelling adults $[34,35]$. Generally, collective social activity is defined as spending time and participating in activities together with other people, such as visiting cultural events, traveling, exercise, or participating in organizational activities. Therefore, social activity requires higher levels of mobility, e.g., more than that required to walk around at home. Moreover, the rate of onset of mobility disabilities after 6 years has been reported to be higher (67.1\%) compared to that of basic ADL disabilities (36.1\%) in patients with depression at baseline [34]. In our study, only a few participants had basic ADL disabilities. Therefore, depressive symptoms likely had a greater impact on high difficulty activities, such as walking long distances, than on easy activities in this study.

In this study, the cut-off values of usual walking speed for predicting ADL difficulty at higher, middle, and lower levels were 83.7, 75.5, and $75.1 \mathrm{~m} / \mathrm{min}$, respectively. These values were much higher compared to values reported previously, possibly because previous studies assessed ADL dependency in community populations. One study reported that usual walking speeds of $1.25 \mathrm{~m} / \mathrm{s}(75.0 \mathrm{~m} / \mathrm{min})$ and $1.07 \mathrm{~m} / \mathrm{s}(64.2 \mathrm{~m} / \mathrm{min})$ predict the onset of basic ADL dependency in men and women, respectively, among a Japanese community population aged 65 to 74 years with no ADL dependency [36]. In order to prevent ADL difficulty, higher physical functioning than that needed to prevent ADL dependency might be required. Therefore, the cut-off values obtained in this study might only be applicable to ambulatory HD outpatients.

Alavi et al. [37] reported that the ADL score is associated with age, gender, and education in hemodialysis and transplant patients. To the best of our knowledge, however, the present study is the first to examine determinants of ADL difficulty related to lower extremities after adjusting for confounders in HD patients. Walking speed and depressive symptoms, both predictors of ADL difficulty, have been reported to improve with adequate exercise training in HD patients $[38,39]$. Thus, exercise 
training may help prevent the onset of ADL difficulty by improving walking speed and depressive symptoms.

This study has some limitations worth noting. First, our study might lack general applicability due to the small sample size and the single-center study design, which included only Japanese HD patients. Second, this study was a cross-sectional study, so a longitudinal study should be carried out in the future to investigate changes in ADL difficulty, physical functions, and depressive symptoms to examine further the determinants of ADL difficulty in HD patients. Third, only CES-D was used to evaluate depressive symptoms. More detailed evaluation including cognitive function will be necessary in the future.

\section{Conclusions}

This cross-sectional study revealed that a slow walking speed and old age are significantly and independently associated with ADL difficulty. The presence of depressive symptoms was significantly and independently associated with ADL difficulty at the higher level of difficulty in ambulatory HD patients. In addition, cut-off values of 83.7, 75.5, and $75.1 \mathrm{~m} / \mathrm{min}$ for usual walking speed predicted the onset of ADL difficulty at higher, middle, and lower levels, respectively.

\section{Additional files}

Additional file 1: Table S1. Twelve items of the questionnaire on perceived mobility difficulty for HD patients. HD, hemodialysis. (XLSX 9 $\mathrm{kb})$

Additional file 2: Table S2. Clinical characteristics, motor function, ADL dependency, and ADL difficulty of study subjects. Data are presented as mean \pm standard deviation or number of subjects. *ADL items with higher level difficulty included "walking up two flights of stairs," "walking $1 \mathrm{~km}$," "walking $600 \mathrm{~m}$," and "rising from the floor." +ADL items with middle level difficulty included "walking down two flights of stairs," "walking 300 m," "walking up one flight of stairs," and "sitting down on the floor." ¥ADL items with lower level difficulty included "walking $20 \mathrm{~m}$ quickly," "walking down one flight of stairs," "walking 100 m," and "rising from a chair." BMI, body mass index: HD, hemodialysis; ADL, activities of daily living. (XLSX $17 \mathrm{~kb}$ )

Additional file 3: Table S3. Univariate and multivariate logistic regression analyses on ADL difficulty. Univariate and multivariate logistic regression analyses were performed with $\mathrm{ADL}$ difficulty as a dependent variable and age, male sex, blood hemoglobin concentration, serum albumin concentration, comorbidity index, depression symptoms, maximum leg strength, one-leg standing time, and usual walking speed as independent variables for each level. *ADL items with higher level difficulty included "walking up two flights of stairs," "walking 1 km," "walking $600 \mathrm{~m}$, " and "rising from the floor." +ADL items with middle level difficulty included "walking down two flights of stairs," "walking 300 m," "walking up one flight of stairs," and "sitting down on the floor." $\neq A D L$ items with lower level difficulty included "walking 20 m quickly," "walking down one flight of stairs," "walking $100 \mathrm{~m}$," and "rising from a chair." ADL, activities of daily living; $\mathrm{OR}$, odds ratio; $\mathrm{Cl}$, confidence interval. (XLSX $15 \mathrm{~kb}$ )

\section{Abbreviations}

ADL: Activities of daily living; AUC: Area under the curve; BMI: Body mass index; CES-D: Center for Epidemiological Studies Depression Screening Index;
Cl: Confidence interval; FIM: Functional Independence Measure HD: Hemodialysis; OR: Odds ratio; ROC: Receiver Operating Characteristic

\section{Acknowledgements}

The authors thank all patients who participated in this study. The authors also thank Drs. Shinya Nakajima, Yoriko Horiguchi, Tomoki Asai, and Sachiko Motohashi for performing the experiments, and the HD treatment staff at the Sagami Circulatory Organ Clinic for their contributions to this study.

\section{Funding}

This research was supported by JSPS KAKENHI Grant Numbers 23500614 and 26350631

\section{Availability of data and materials}

We decided not to share the data in our study because all data are thoroughly described and reflected in the accompanying tables and figures (all relevant data are within the paper).

\section{Authors' contributions}

TW, TK, NM, YM, RM, YT, AY, and AM analyzed and interpreted the patient data regarding $A D L$ difficulty and contributed significantly to the preparation of the manuscript. $\mathrm{KY}, \mathrm{MH}$, and TS performed measurements of motor function, depressive symptom, ADL dependency, and ADL difficulty. All authors read and approved the final manuscript.

\section{Ethics approval and consent to participate}

This study was approved by the Ethics Committee of Kitasato University School of Allied Health Sciences and was conducted in accordance with the standards set forth by the latest revision of the Declaration of Helsinki. All patients received a detailed explanation of the study protocol and provided informed consent.

\section{Consent for publication}

Not applicable.

\section{Competing interests}

The authors declare that they have no competing interests.

\section{Publisher's Note}

Springer Nature remains neutral with regard to jurisdictional claims in published maps and institutional affiliations.

\section{Author details}

'Department of Rehabilitation Sciences, Kitasato University Graduate School of Medical Sciences, 1-15-1 Kitasato, Sagamihara, Kanagawa 252-0373, Japan. ${ }^{2}$ Department of Physical Therapy, School of Health Sciences, Tokyo University of Technology, 5-23-22 Nishikamata, Ota-ku, Tokyo 144-8535, Japan. ${ }^{3}$ Department of Sleep Medicine, Kitasato University Graduate School of Medical Sciences, 1-15-1 Kitasato, Sagamihara, Kanagawa 252-0373, Japan ${ }^{4}$ School of Allied Health Sciences, Kitasato University, 1-15-1 Kitasato, Sagamihara, Kanagawa 252-0373, Japan. ${ }^{5}$ Department of Rehabilitation, Kitasato University Hospital, 1-15-1 Kitasato, Sagamihara, Kanagawa 252-0375, Japan. ${ }^{6}$ Department of Nephrology in Internal Medicine, Kitasato University School of Medicine, 1-15-1 Kitasato, Sagamihara, Kanagawa 252-0373, Japan. ${ }^{7}$ Department of Hemodialysis Center, Sagami Circulatory Organ Clinic, 4-21-15 Sonan, Sagamihara, Kanagawa 252-0312, Japan.

Received: 14 September 2017 Accepted: 10 January 2018

Published online: 07 February 2018

\section{References}

1. National Kidney Foundation. K/DOQI clinical practice guidelines: cardiovascular disease in dialysis patients. Am J Kidney Dis. 2005;45(4 Suppl 3) https://doi.org/10.1053/j.ajkd.2005.01.018.

2. Farrington K, Covic A, Aucella F, Clyne N, de Vos L, Findlay A, et al. Clinical practice guideline on management of older patients with chronic kidney disease stage 3b or higher (eGFR $<45 \mathrm{~mL} / \mathrm{min} / 1.73 \mathrm{~m} 2$ ). Nephrol Dial Transplant. 2016;31(Suppl 2):ii1-ii66. https://doi.org/10.1093/ndt/gfw356.

3. Painter P, Marcus RL. Assessing physical function and physical activity in patients with CKD. Clin J Am Soc Nephrol. 2013;8(5):861-72. https://doi.org/ 10.2215/CJN.06590712. 
4. Matsuzawa R, Matsunaga A, Wang G, Yamamoto S, Kutsuna T, Ishii A. Relationship between lower extremity muscle strength and all-cause mortality in Japanese patients undergoing dialysis. Phys Ther. 2014;94(7): 947-56. https://doi.org/10.2522/ptj.20130270.

5. Altintepe L, Levendoglu F, Okudan N, Guney I, Savas Cilli A, Ugurlu H, et al. Physical disability, psychological status, and health-related quality of life in older hemodialysis patients and age-matched controls. Hemodial Int. 2006; 10(3):260-6. https://doi.org/10.1111/j.1542-4758.2006.00106.X.

6. Kutner NG, Zhang R, Huang Y, Painter P. Gait speed and mortality, hospitalization, and functional status change among hemodialysis patients: a US renal data system special study. Am J Kidney Dis. 2015;66(2):297-304. https://doi.org/10.1053/j.ajkd.2015.01.024.

7. Jassal SV, Karaboyas A, Comment LA, Bieber BA, Morgenstern H, Sen A, et al. Functional Dependence and Mortality in the International Dialysis Outcomes and Practice Patterns Study (DOPPS). Am J Kidney Dis. 2016;67(2): 283-92. https://doi.org/10.1053/j.ajkd.2015.09.024

8. Kutner NG, Brogan DJ. Assisted survival, aging, and rehabilitation needs: comparison of older dialysis patients and age-matched peers. Arch Phys Med Rehabil. 1992;73(4):309-15. https://doi.org/10.1016/00039993(92)90001-D.

9. Kaysen GA, Larive B, Painter P, Craig A, Lindsay RM, Rocco MV, et al. Baseline physical performance, health, and functioning of participants in the Frequent Hemodialysis Network (FHN) trial. Am J Kidney Dis. 2011;57(1): 101-12. https://doi.org/10.1053/j.ajkd.2010.08.021.

10. van den Ham EC, Kooman JP, Schols AM, Nieman FH, Does JD, Franssen FM, et al. Similarities in skeletal muscle strength and exercise capacity between renal transplant and hemodialysis patients. Am J Transplant. 2005; 5(8):1957-65. https://doi.org/10.1111/j.1600-6143.2005.00944.x.

11. Fahal $I H$, Bell GM, Bone JM, Edwards RH. Physiological abnormalities of skeletal muscle in dialysis patients. Nephrol Dial Transplant. 1997;12(1):11927. https://doi.org/10.1093/ndt/12.1.119.

12. Painter P, Carlson L, Carey S, Paul SM, Myll J. Physical functioning and health-related quality-of-life changes with exercise training in hemodialysis patients. Am J Kidney Dis. 2000;35(3):482-92. https://doi.org/10.1016/S02726386(00)70202-2

13. Lopes AA, Albert JM, Young EW, Satayathum S, Pisoni RL, Andreucci VE, et al. Screening for depression in hemodialysis patients: associations with diagnosis, treatment, and outcomes in the DOPPS. Kidney Int. 2004;66(5): 2047-53. https://doi.org/10.1111/j.1523-1755.2004.00977.x

14. Chaves PH, Garrett ES, Fried LP. Predicting the risk of mobility difficulty in older women with screening nomograms: the Women's Health and Aging Study II. Arch Intern Med. 2000;160(16):2525-33. https://doi.org/10.1001/ archinte.160.16.2525.

15. Fried LF, Lee JS, Shlipak M, Chertow GM, Green C, Ding J, et al. Chronic kidney disease and functional limitation in older people: health, aging and body composition study. J Am Geriatr Soc. 2006;54(5):750-6. https://doi.org/ 10.1111/j.1532-5415.2006.00727.x.

16. Connolly D, Garvey J, McKee G. Factors associated with ADL/IADL disability in community dwelling older adults in the lrish longitudinal study on ageing (TILDA). Disabil Rehabil. 2017;39(8):809-16. https://doi.org/10.3109/ 09638288.2016 .1161848 .

17. Tomita A, Burns JK. Depression, disability and functional status among community-dwelling older adults in South Africa: evidence from the first South African National Income Dynamics Study. Int J Geriatr Psychiatry. 2013;28(12):1270-9. https://doi.org/10.1002/gps.3954.

18. Hirvensalo M, Rantanen T, Heikkinen E. Mobility difficulties and physical activity as predictors of mortality and loss of independence in the community-living older population. J Am Geriatr Soc. 2000;48(5):493-8. https://doi.org/10.1111/j.1532-5415.2000.tb04994.x

19. Liu J, Huang Z, Gilbertson DT, Foley RN, Collins AJ. An improved comorbidity index for outcome analyses among dialysis patients. Kidney Int. 2010;77(2):141-51. https://doi.org/10.1038/ki.2009.413.

20. Irwin $\mathrm{M}$, Artin $\mathrm{KH}$, Oxman MN. Screening for depression in the older adult: criterion validity of the 10-item Center for Epidemiological Studies Depression Scale (CES-D). Arch Intern Med. 1999;159(15):1701-4. https://doi. org/10.1001/archinte.159.15.1701.

21. Andresen EM, Malmgren JA, Carter WB, Patrick DL. Screening for depression in well older adults: evaluation of a short form of the CES-D (Center for Epidemiologic Studies Depression Scale). Am J Prev Med. 1994;10(2):77-84.

22. Kutsuna T, Matsunaga A, Matsumoto T, Ishii A, Yamamoto $K$, Hotta $K$, et al. Physical activity is necessary to prevent deterioration of the walking ability of patients undergoing maintenance hemodialysis. Ther Apher Dial. 2010; 14(2):193-200. https://doi.org/10.1111/j.1744-9987.2009.00750.x.

23. Bohannon RW. Comfortable and maximum walking speed of adults aged 20-79 years: reference values and determinants. Age Ageing. 1997;26(1):159. https://doi.org/10.1093/ageing/26.1.15.

24. Yamamoto S, Matsunaga A, Kamiya K, Miida K, Ebina Y, Hotta K, et al. Walking speed in patients with first acute myocardial infarction who participated in a supervised cardiac rehabilitation program after coronary intervention. Int Heart J. 2012;53(6):347-52. https://doi.org/10.1536/ihj.53.347.

25. Michikawa T, Nishiwaki Y, Takebayashi T, Toyama Y. One-leg standing test for elderly populations. J Orthop Sci. 2009;14(5):675-85. https://doi.org/10. 1007/s00776-009-1371-6.

26. Hinkle JL. The use of the FIM ${ }^{\top M}$ instrument in the poststroke population. Top Stroke Rehabil. 2000;7(3):47-66. https://doi.org/10.1310/3LB6-CGVN-EAAUOTEQ.

27. Granger CV, Hamilton BB, Keith RA, Zielezny M, Sherwin F. Advances in functional assessment for medical rehabilitation. Top Geriatr. 1986;1(3):5974. https://doi.org/10.1097/00013614-198604000-00007.

28. Ottenbacher KJ, Hsu Y, Granger CV, Fiedler RC. The reliability of the functional independence measure: a quantitative review. Arch Phys Med Rehabil. 1966; 77(12):1226-32. https://doi.org/10.1016/S0003-9993(96)90184-7.

29. Ozawa T, Kutsuna T, Matsushima S, Kobayashi S, Henmi F, Matsuzawa R, et al. Development of a questionnaire for assessing perceived difficulty in performing activities of daily living related to lower-limb function in ambulatory hemodialysis patients (in Japanese). Phys Ther Jpn. 2010; 37(1):9-16.

30. Ozawa T, Matsunaga A, Kutsuna T, Matsushima S, Kobayashi S, Matsuzawa R, et al. Development of a questionnaire for assessing perceived difficulty in performing activities of daily living related to lower-limb function in ambulatory hemodialysis patients. Assessment of the responsiveness to exercise training (in Japanese). J Jpn Soc Dial Ther. 2010;43(6):515-22. https://doi.org/10.4009/jsdt.43.515.

31. Perkins NJ, Schisterman EF. The inconsistency of "optimal" cutpoints obtained using two criteria based on the receiver operating characteristic curve. Am J Epidemiol. 2006;163(7):670-5. https://doi.org/10.1093/aje/ kwj063.

32. Dunlay SM, Manemann SM, Chamberlain AM, Cheville AL, Jiang R, Weston SA, et al. Activities of daily living and outcomes in heart failure. Circ Heart Fail. 2015;8(2):261-7. https://doi.org/10.1161/CIRCHEARTFAILURE.114.001542.

33. Capistrant BD, Glymour MM, Berkman LF. Assessing mobility difficulties for cross-national comparisons: results from the World Health Organization Study on Global AGEing and Adult Health. JAGS. 2014;62(2):329-35. https:// doi.org/10.1111/jgs.12633.

34. Penninx BW, Leveille S, Ferrucci L, van Eijk JT, Guralnik JM. Exploring the effect of depression on physical disability: longitudinal evidence from the established populations for epidemiologic studies of the elderly. Am J Public Health. 1999;89(9):1346-52.

35. Djernes JK. Prevalence and predictors of depression in populations of elderly: a review. Acta Psychiatr Scand. 2006;113(5):372-87. https://doi.org/ 10.1111/j.1600-0447.2006.00770.x.

36. Shinkai S, Watanabe S, Kumagai S, Fujiwara Y, Amano H, Yoshida H, et al. Walking speed as a good predictor for the onset of functional dependence in a Japanese rural community population. Age Ageing. 2000;29(5):441-446. doi:doi.org/10.1093/ageing/29.5.441.

37. Alavi NM, Aliakbarzadeh Z, Sharifi K. Depression, anxiety, activities of daily living, and quality of life scores in patients undergoing renal replacement therapies. Transplant Proc. 2009:41(9):3693-6. https://doi.org/10.1016/j. transproceed.2009.06.217.

38. Headley S, Germain M, Mailloux P, Mulhern J, Ashworth B, Burris J, et al. Resistance training improves strength and functional measures in patients with end-stage renal disease. Am J Kidney Dis. 2002:40(2):355-64. https:// doi.org/10.1053/ajkd.2002.34520.

39. Carney RM, Templeton B, Hong BA, Harter HR, Hagberg JM, Schechtman KB, et al. Exercise training reduces depression and increases the performance of pleasant activities in hemodialysis patients. Nephron. 1987;47(3):194-8. https://doi.org/10.1159/000184490. 